\title{
Depletion of nonrenewable energy resources, entropy crisis and nanotechnology solutions
}

The steady depletion of global energy resources due to increased consumption by mankind has contributed to the severe problem of exhausting all available non renewable energy resources such as natural gas, oil and coal. It is easy to do a simple calculation and forecast that global non-renewable energy resources will be exhausted within the next 50 years, if the present rate of consumption of these resources continues. The obvious question arising from the above is 'how should the world respond in order to solve this important problem of depletion of non renewable energy resources?' The obvious answers to this question lies in the development of alternative energy resources, which are renewable,reduction of consumption of the presently used energy resources and/ or supplementing them with alternative means to prolong their use.

At the outset, it is important to understand that there is no energy crisis in the universe according to the 'Law of Conservation of Energy'. What contributes to the crisis is entropy, i.e. dispersal of energy. Since the tendency for natural processes to occur is in the direction of increasing entropy (randomness, dispersal of energy) of the universe, energy is continuously being dispersed making it increasingly difficult for energy to be harvested for useful work. All combustion reactions which provide useful energy for human needs are exothermic and generate more gaseous products leading to an increase in entropy. Both these factors contribute favourably to spontaneity (natural direction) of these reactions. As such, reversal of combustion reactions requires a considerable amount of energy. This makes coal, fossil fuels, wood and petroleum fuel resources virtually non-renewable in the realistic time scale.

Is the nuclear energy a feasible solution to this problem? It is indeed a short-term solution since the associated risks and threats might surpass its usefulness. Nevertheless, the nuclear fusion reactions taking place in the sun does not have any adverse effect on the Earth's Biosphere. The Earth receives, on average, solar energy at a steady rate of 2.4 kilojoules per square centimetre during day time, in the virtually harmless near ultraviolet, entire visible and near infrared regions. This energy supply is virtually inexhaustible. Therefore, it is important to design and develop efficient solar energy conversion devices which would help solve the greatest threat ahead of us. In doing so, they must analyse all possible means of constructing efficient solar energy conversion devices. Taking lessons from nature would be very important in this context.

The natural photosynthetic system that harnesses solar energy and converts carbon dioxide and water into carbohydrates and oxygen is a marvellous arrangement of molecular and supramolecular assemblies which favour the complex processes associated with photosynthesis which occurs, mainly, in plants. The major processes of photosynthesis are conversion of solar energy into electricity and storage of the resulting electricity in chemical bonds of sugar molecules. The overall chemical reaction:

$$
6 \mathrm{CO}_{2}(\mathrm{aq})+6 \mathrm{H}_{2} \mathrm{O}(\mathrm{l}) \stackrel{\mathrm{h} v}{\longrightarrow} \mathrm{C}_{6} \mathrm{H}_{12} \mathrm{O}_{6}(\mathrm{~s})+6 \mathrm{O}_{2}(\mathrm{~g})
$$

is energy-wise unfavourable and, hence, there is no natural tendency to proceed unless the required energy is supplied externally. The current understanding of the mechanism of photosynthesis is that it involves three series of interconnected oxidation-reduction reactions. The first is the evolution of oxygen from water yielding hydrogen atoms (Photosystem II). The second is the transfer of hydrogen atoms to a primary hydrogen acceptor and the third is the reduction of carbon dioxide to carbohydrates by the primary hydrogen acceptor (Photosystem I). The light energy required for photosynthesis is used to drive the hydrogen atoms against the potential gradient. The two photo reactions take place in two pigment systems - Photosystems I and II - interconnected by enzymatic reactions coupled with photophosphorylation yielding adenosine triphosphate (ATP). ATP is one of the high energy $(30 \mathrm{~kJ}$ liberated 
upon hydrolysis per mole) compounds used in biological systems for chemical energy storage.

Undoubtedly, an artificial photosynthetic system should have molecular and supramolecular assemblies like those arranged in the natural photosynthetic system. Such systems must be capable of absorbing solar energy directed onto the Earth, separating the resulting charges, protecting the charges from various losses and transporting those to catalytic sites where the above mentioned half reactions become feasible. The global research efforts devoted to develop such systems and amount of money spent, on these activities in the last two decades are far too much to account in terms of realistic finite figures compared to actual success which is very small. However, nano-structured inorganic semiconductor hetero-junction solar cells and nanostructured dye-sensitized solar cells stand out as somewhat successful stories. important nanoscale architecture in the areas of lightinduced electron transfer chemistry and solar energy conversion devices. Recent synthetic advances have contributed to the development of functionalised fullerenes which could be covalently linked with other molecules. The robustness and negligible change of structure due to electronic excitations and transfers guarantee the long term stability of fullerenes as electron acceptors in solar energy conversion devices. Carbon nanotubes (CNTs) share structural features common with fullerenes but are cylindrical in shape. The single cylinder type of rolled graphene sheets are called single-walled carbon nanotubes (SWCNTs) and those with several cocentric cylinders one placed within another forming a bundle of tubes are called multi-walled carbon nanotubes (MWCNTs). An SWCNT has exceptionally high tensile strength and is the material known so far to have the highest thermal conductivity and electronic conductivity exceeds that of silver and copper metals in the axial

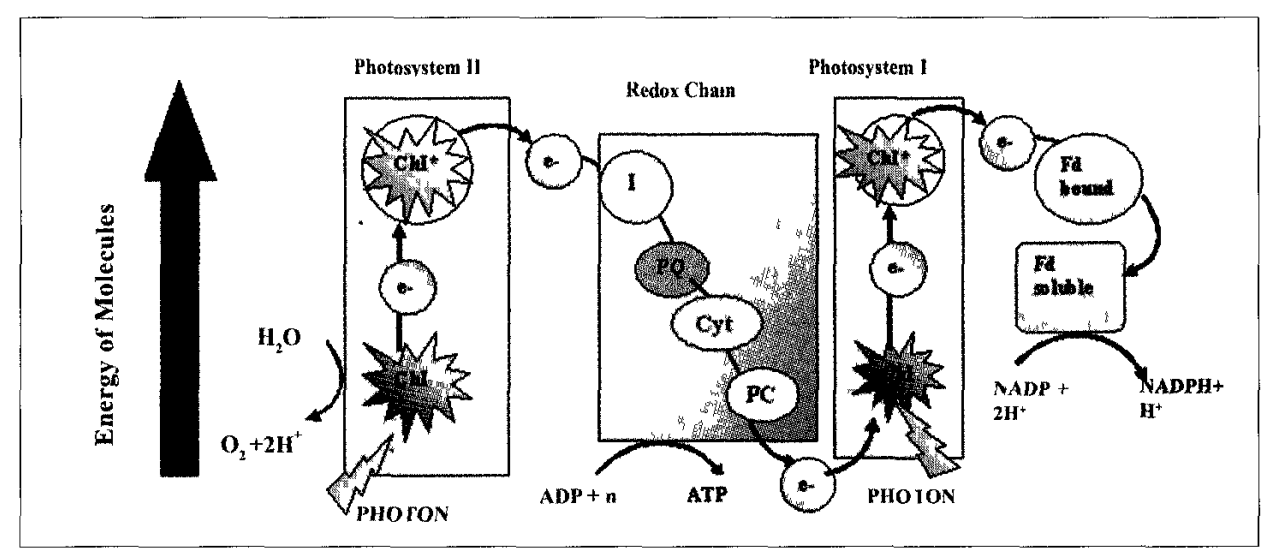

Figure 1: Overall processes associated with photosynthesis

As we can see, nature induces problems for which solutions are there in nature itself. If we can unveil the valuable hidden secrets of nature, we can find solutions for the problems that we are faced with. It is the trained intelligent mind that is able to unveil the nature's hidden secrets that can serve as solutions to these problems. Further, accidental discoveries are blessings in disguise. In fact, the majority of important discoveries belong to this category. Curiosity of intelligent minds help uncover nature's hidden treasures. Two such recently discovered treasures are the so called 'Buckminster fullerenes' and 'carbon nanotubes'. The most common structures of fullerenes constitute 60 carbon atoms joined in the shape of a football. These structures comprise electron-rich hexagons and electron-deficient pentagons with extraordinary electron acceptor properties. $\mathrm{AC}_{60}$ molecule has a diameter of $7.8 \AA(=0.78 \mathrm{~nm})$ and represents direction but has virtually zero conduction in the lateral and radial directions. Thus, it not only exhibits extraordinary electron accepting properties but also provides high fields at the donor/CNT interfaces for exciton dissociation. Both these properties are useful for solar energy conversion devices. The CNT also has favourable field emission properties useful in fabricating Light Emitting Diodes (LED).

As far as light absorbing properties are concerned, both fullerenes and CNTs are only moderate absorbers. It would be ideal to have a separate unit to absorb light as in the natural system and. to utilize porphyrins and phthalocyanins to fulfil this task. Indeed several porphyrin and phthalocyanin derivatives have already been coupled chemically through covalent linkages to fullerenes and CNTs, and the resulting supramolecular assemblies have 
been utilised in solar cells. These materials hold great promise as integrative building blocks, with increased absorption cross-section in the visible region and very high electronic excitation energy exceeding $2.0 \mathrm{eV}$ which can power the conversion of light energy into chemical or electrical energy, in the design of artificial photosynthetic systems.

Zinc porphyrin derivatives covalently linked to fullerenes have been covalently linked with conducting indium doped tin oxide electrodes to be used as photoelectrodes. The cells fabricated with such nanoarchitecture have promising photon-to-photocurrent conversion efficiencies. When de-metallated porphyrin peptide octamerand $\mathrm{C}_{60}$ was used, high power conversion efficiency of $1.3 \%$ and incident photon-to-photocurrent conversion efficiency of $42 \%$ have been realised.
Photoelectrochemical solar cells constructed using nanoarchitectures consisting of SWCNT and porphyrin moieties exhibit incident photon-to-photocurrent conversion efficiency of $13 \%$ at $0.2 \mathrm{~V}$ potential bias versus the saturated calomel electrode. These systems do mimic the natural photosynthetic system and show some promise in converting solar energy to chemical or electrical energy.

The recently discovered allotropes of carbon, which have dimensions in the nanometre range, stand out to be the most promising materials to be used in finding a solution to this severe problem faced by mankind. Until the next amazing new treasure is unveiled, the world today is gifted with fullerenes and carbon nanotubes to design, device and fabricate useful tools taking lessons from nature.

\section{R.M.G. Rajapakse}

Department of Chemistry, University of Peradeniya, Peradeniya. 